Pacific Journal of Mathematic 


\title{
ISOMORPHISM ORDER FOR ABELIAN GROUPS
}

\author{
STEVEN BRYANT
}

In the theory of isometric embedding in metric spaces the following theorem is proved: Let $M$ be a metric space every $n+3$ points of which can be mapped isometrically into Euclidean $n$-space, then there exists an isometry from all of $M$ into Euclidean $n$-space. Because of this theorem Euclidean $n$-space is said to have congruence order $n+3$. [1].

L. M. Blumenthal has raised the question as to whether a notion analogous to that of congruence order could be developed for algebraic systems. In this paper a definition of isomorphism order is introduced for groups and a complete description of all Abelian groups having finite or hyperfinite isomorphism order is obtained.

First a well known definition to avoid any possible misunderstanding of the use of the concept of rank.

Definition. A group $G$ is said to have rank $n$ if every finitely generated subgroup can be generated by $n$ or fewer elements and $n$ is the smallest natural number with this property.

For convenience we introduce the following definition.

Definition. If $k$ elements $g_{1}, g_{2}, \cdots, g_{k}$ of a group $G$ generate a subgroup of $G$ which is isomorphic to a subgroup of a group $H$, we will say that $g_{1}, g_{2}, \cdots, g_{k}$ are embeddable in $H$ and that the subgroup generated by the $g$ 's is embeddable in $H$.

Now we are ready for the definition of isomorphism order.

DEFINITION. A group $G$ is said to have isomorphism order $k$ if and only if any group $H$ is embeddable in $G$ whenever every $k$ of its elements are embeddable in $G$.

In the above definition $k$ may be any cardinal number, however, in this paper $k$ will always stand for a natural number.

If $A$ and $B$ are two cardinal numbers such that $A$ is less than or equal to $B$ then it is easy to see that if a group $G$ has isomorphism order $A$ then $G$ has isomorphism order $B$.

Every group has some isomorphism order, since if $G$ is a group of cardinality $M$ then $G$ has isomorphism order $N$ where $N$ is any cardinal

Presented to the Society November 17, 1956; received by the editors May 14, 1958, 
number which is larger than $M$. Since the cardinals can be well ordered every group has a smallest isomorphism order. However, in what is to follow, if we say $G$ has isomorphism order $k$ we will not mean that $k$ is the smallest isomorphism order of $G$ unless we explicitly say so.

The following lemmas lead to a theorem describing all Abelian groups having finite isomorphism order.

Lemma 1. Let $k$ be a natural number and $p$ a fixed prime. Let $G$ be a direct sum of. $k$ groups each of. which is a cyclic group of order a power of $p$ or a group isomorphic to $Z(P \infty)$. Then $G$ has isomorphism order $k+1$.

Proof. Let $H$ be a group every $k+1$ elements of which are embeddable in $G$. $H$ is primary and has rank $k$. From this the conclusion easily follows. (Exercise 49, [2])

Lemma 2. An Abelian torsion group $G$ has isomorphism order $k$ if and only if $G$ is a direct sum of fewer than $k$ subgroups of the rationals mod one.

Proof. Let $G$ be an Abelian torsion group having isomorphism order $k$. Write $G$ as a direct sum of primary groups that is $G=\sum G_{p}$, where $p$ ranges over the primes and $G_{p}$ consists of all elements whose order is a power of $p$. Now $G_{p}$ does not contain the integers mod $p$ taken $k$ times for, if it did, arbitrarily large groups constructed by taking direct sums of the integers mod $p$ would (by hypothesis) be embeddable in $G$. From this it follows that $G_{p}$ has rank less than $k$. Hence (exercise $49,[2]) G_{p}$ is a direct sum of fewer than $k$ subgroups of $Z(P \infty)$, and therefore $G$ is a direct sum of fewer than $k$ subgroups of the rationals mod one by rearrangement of summands.

Conversely, let, $G$ be a direct sum of fewer than $k$ subgroups of the rationals mod one. Let $H$ be a group every $k$ elements of which are embeddable in $G$, so that $H$ is torsion. Write $H=\sum H_{p}$ and consider $H_{p}$. Every $k$ elements of $H_{p}$ are embeddable in $G_{p}$, but by Lemma 1 , $G_{p}$ has isomorphism order $k$, hence $H_{p}$ is embeddable in $G_{p}$ and so $H$ is embeddable in $G$.

Lemma 3. A torsion free Abelian group has isomorphism order $k$ if and only if it is a vector space over the rationals of dimension less than $k$.

Proof. Let $G$ be a torsion free Abelian group having isomorphism order $k$. Now $G$ does not contain the direct sum of the integers taken $k$ times, for, if it did, the group consisting of the direct sum of the 
integers taken a greater number of times than the cardinality of $G$ would have every $k$ elements embeddable in $G$ and hence by hypothesis would be embeddable in $G$, a contradiction.

Let $m$ be the maximal number of elements of $G$ which are independent over the integers. By what was just said $m$ must be less than $k$. Any $m$ dimensional vector space over the rationals is embeddable in $G$, by hypothesis. So $G$ contains a vector space over the rationals of dimension $\mathrm{m}$, call this space $V$. The space $V$ is a divisible subgroup of $G$ and hence is a direct summand so $G=A+V$. Let a be a nonzero element of $A$. Since $m$ is the maximal number of independent elements of $G, n a$ is in $V$ for some nonzero integer $n$, but since $n a$ is in $A$ it is zero and therefore $a$ is zero and so $G=V$.

Conversely, if $G$ is a vector space over the rationals of dimension less than $k$ and $H$ is a group every $k$ elements of which are embeddable in $G$ then $H$ is embeddable in $G$. To see this, observe that $H$ can be embedded in a vector space over the rationals consisting of all couples of the form $(n, h$,$) when n$ is a nonzero integer and equivalence is defined in the natural way, and the dimension of this space is less than $k$ for if not, there exist $k$ elements of $H$ not embeddable in $G$, which completes the proof.

Theorem 1. An Abelian group $G$ has isomorphism order $k$ if and only if $G$ is the direct sum of two groups, one torsion, the other torsion free. The torsion free group is a vector space over the rationals of dimension less than $k$, while the torsion group can be written as a direct sum of fewer than $k$ subgroups of the rationals mod one.

Proof. Let $G$ be an Abelian group having isomorphism order $k$. The theorem follows from the lemmas if $G$ is torsion or torsion free. Now $G$ contains a vector space $V$ over the rationals of dimension $n$ less than $k$ where $n$ is the maximal number of elements of $G$ which are independent over the integers. This holds by an application of the argument of Lemma 3. Regard $V$ as a group, then $V$ is a direct summand of $G$ since $V$ is divisible. So $G=A+V$ and $A$ is torsion, for if $x$ is in $A$ then $m x$ is in $V$ for some nonzero integer $m$, hence $m x=0$. Now apply Lemma 2 to $A$ and obtain the necessity of the theorem.

To prove the sufficiency, let $G$ be an Abelian group such that $G=$ $T+V$ where $T=A_{1}+A_{2}+\cdots+A_{s}$ and each $A_{i}$ is a subgroup of the rationals mod one and $s<k$, and $V$ is a vector space over the rationals of dimension less than $k$.

We must show that if $H$ is an Abelian group, every $k$ (or fewer) elements of which are embeddable in $G$, then $H$ is embeddable in $G$.

$H$ does not contain $k$ elements which are independent over the 
integers. Hence $H$ contains at least one subgroup $H_{0}$ such that $h \in H$ implies $r h \in H_{0}$ for some natural number $r$ and such that $H_{0}$ is embeddable in $G$.

Let $T^{*}$ be the direct sum of the rationals mod one taken $s$ times. Let $G^{*}=T^{*}+V$. We will show that if $\phi$ is an isomorphism from $H_{\text {J }}$ into $G^{*}$ then if $H_{0} \neq H, \phi$ can be proporly extended. Then the embeddability of $H$ in $G^{*}$ can be obtained by a transfinite argument. Finally, we will see that $H$ is embeddable in $G$.

So let $H_{0}$ be a subgroup of $H$ such that $h \in H$ implies $r h \in H_{0}$ for some integer $r$ and let $F$ be an isomorphism from $H_{0}$ into $G^{*}$. If $H_{0}=$ $H$ we are done, if not, let $h \notin H_{0}$, and $m$ the smallest natural number such that $m h \in H_{0}$.

Case 1. $m=p, p$ a prime. Let $M=\left[z \mid p z=F(p h), z \in G^{*}\right]$. For convenience, we will refer to $M$ as the set of all the "pth roots" of $F(p h)$, and note that $M$ is finite, and that the number of elements in $M$ is exactly the number of " $p$ th roots" of 0 in $G^{*}$. Now, not every element of $M$ is in $F\left(H_{0}\right)$, for if so, a glance at the inverse images will show that the inverse image of every element of $M$ is a " $p$ th root" of $p h$. But $F(p h)$ has at least as many " $p$ th roots" in $G^{*}$ as $p h$ has in $H$. Hence $h$ itself is in $H_{0}$ a contradiction.

We conclude that some element of $M$, call it $z$, is not in $F\left(H_{0}\right)$. Furthermore, if $0<n<p$, then $n z \notin F\left(H_{0}\right)$ and hence $F$ can be extended in the natural way.

Case 2. $m$ not a prime, then $m=q t$ where $q$ is a prime. Apply the argument of Case 1 to the set of all $q$ th roots of $F(m h)$.

This shows that $H$ is embeddable in $G^{*}$. But by Lemma 2, if $T^{\prime}$ is the torsion subgroup of $H, T^{\prime}$ is embeddable in $T$. Hence it is easily seen that $H$ is actually embeddable in $G$, which completes the proof.

In the above theorem, nothing has been said about smallest isomorphism order. However, it is easy to see that, if $G$ has smallest isomorphism order $k$ then either the torsion free summand of $G$ has rank $k-1$ or the torsion summand cannot be written as a direct sum of fewer than $k-1$ subgroups of the rationals mod 1 .

The next step up in the hierarchy of isomorphism order is given by the following definition.

DeFINITION. A group $G$ is said to have hyperfinite isomorphism order if, whenever every finitely generated subgroup of a group $H$ is embeddable in $G$, then $H$ is embeddable in $G$.

The proof of the next theorem is similar to that of Theorem 1, and 
rests on the fact that a torsion group has hyperfinite isomorphism order if and only if the rank of each primary subgroup is finite, while a torsion free group has hyperfinite isomorphism order if it is a finite dimensional vector space over the rationals.

THEOREM 2. An Abelian group $G$ has hyperfinite isomorphism order if and only if it is the direct sum of two groups, one torsion, the other torsion free. The torsion free group is a finite dimensional vector space over the rationals while the torsion summand has no primary subgroup of infinite rank.

REMARK. If the smallest isomorphism order $G$ has is hyperfinite, then there is no upper bound on the ranks of the primary subgroups of $G$.

This concludes the analysis of Abelian groups having finite or hyperfinite isomorphism order. ${ }^{2}$ In a subsequent paper, we hope to give some results concerning Abelian groups having transfinite isomorphism order. Also, this notion can be carried over to other systems, such as rings, a direction in which some preliminary results have been obtained.

\section{REFERENCES}

1. L. M. Blumenthal, Theory and applications of distance geometry, Oxford at the Clarendon Press, 1953.

2. I. Kaplansky, Infinite Abelian groups, University of Michigan Press, Ann Arbor, 1954.

Fresno State College

UNIVERSITY OF MISSOURI

2 The author wishes to acknowledge his indebtedness to Joseph Zemmer for help in the initial stages of this work, and to Paul Cohen for part of the proof of Theorem 1. 



\section{PACIFIC JOURNAL OF MATHEMATICS}

\section{EDITORS}

\section{David Gilbarg}

Stanford University

Stanford, California

\section{R. A. Beaumont}

University of Washington

Seattle 5, Washington

\author{
A. L. Whiteman
}

University of Southern California Los Angeles 7, California

E. G. Straus

University of California

Los Angeles 24, California

\section{ASSOCIATE EDITORS}

\author{
E. F. BECKENBACH \\ C. E. BURGESS \\ M. HALL \\ E. HEWITT
}
A. HORN
V. GANAPATHY IYER
R. D. JAMES
M. S. KNEBELMAN
L. NACHBIN
I. NIVEN
T. G. OSTROM
H. L. ROYDEN

M. M. SCHIFFER

G. SZEKERES

F. WOLF

K. YOSIDA

\section{SUPPORTING INSTITUTIONS}

\author{
UNIVERSITY OF BRITISH COLUMBIA \\ CALIFORNIA INSTITUTE OF TECHNOLOGY \\ UNIVERSITY OF CALIFORNIA \\ MONTANA STATE UNIVERSITY \\ UNIVERSITY OF NEVADA \\ OREGON STATE COLLEGE \\ UNIVERSITY OF OREGON \\ OSAKA UNIVERSITY \\ UNIVERSITY OF SOUTHERN CALIFORNIA
}

\author{
STANFORD UNIVERSITY \\ UNIVERSITY OF TOKYO \\ UNIVERSITY OF UTAH \\ WASHINGTON STATE COLLEGE \\ UNIVERSITY OF WASHINGTON \\ * * * * \\ AMERICAN MATHEMATICAL SOCIETY \\ CALIFORNIA RESEARCH CORPORATION \\ HUGHES AIRCRAFT COMPANY \\ THE RAMO-WOOLDRIDGE CORPORATION
}

Mathematical papers intended for publication in the Pacific Journal of Mathematics should be typewritten (double spaced), and the author should keep a complete copy. Manuscripts may be sent to any one of the four editors. All other communications to the editors should be addressed to the managing editor, E. G. Straus at the University of California, Los Angeles 24, California.

50 reprints per author of each article are furnished free of charge; additional copies may be obtained at cost in multiples of 50 .

The Pacific Journal of Mathematics is published quarterly, in March, June, September, and December. The price per volume (4 numbers) is $\$ 12.00$; single issues, $\$ 3.50$. Back numbers are available. Special price to individual faculty members of supporting institutions and to individual members of the American Mathematical Society: $\$ 4.00$ per volume; single issues, $\$ 1.25$.

Subscriptions, orders for back numbers, and changes of address should be sent to Pacific Journal of Mathematics, 2120 Oxford Street, Berkeley 4, California.

Printed at Kokusai Bunken Insatsusha (International Academic Printing Co., Ltd.), No. 6, 2-chome, Fujimi-cho, Chiyoda-ku, Tokyo, Japan.

PUBLISHED BY PACIFIC JOURNAL OF MATHEMATICS, A NON-PROFIT CORPORATION

The Supporting Institutions listed above contribute to the cost of publication of this Journal, but they are not owners or publishers and have no responsibility for its content or policies. 


\section{Pacific Journal of Mathematics}

\section{Vol. 8, No. $4 \quad$ June, 1958}

Richard Arens, The maximal ideals of certain functions algebras ........ 641

Glen Earl Baxter, An operator identity ........................... 649

Robert James Blattner, Automorphic group representations ........... 665

Steve Jerome Bryant, Isomorphism order for Abelian groups ............ 679

Charles W. Curtis, Modules whose annihilators are direct summands...... 685

Wilbur Eugene Deskins, On the radical of a group algebra ............ 693

Jacob Feldman, Equivalence and perpendicularity of Gaussian

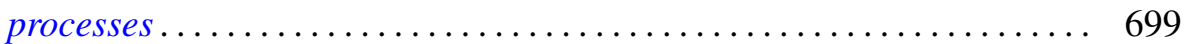

Marion K. Fort, Jr. and G. A. Hedlund, Minimal coverings of pairs by triples....................................... 709

I. S. Gál, On the theory of $(m, n)$-compact topological spaces ......... 721

David Gale and Oliver Gross, A note on polynomial and separable games........................................ 735

Frank Harary, On the number of bi-colored graphs ............... 743

Bruno Harris, Centralizers in Jordan algebras ................... 757

Martin Jurchescu, Modulus of a boundary component ............... 791

Hewitt Kenyon and A. P. Morse, Runs . . . . . . . . . . . . . . . . . . . . . . 811

Burnett C. Meyer and H. D. Sprinkle, Two nonseparable complete metric

spaces defined on $[0,1] \ldots \ldots \ldots \ldots \ldots \ldots \ldots \ldots \ldots \ldots \ldots . \ldots . \ldots . \ldots . \ldots 25$

M. S. Robertson, Cesàro partial sums of harmonic series expansions...... 829

John L. Selfridge and Ernst Gabor Straus, On the determination of numbers by their sums of a fixed order ........................ 847

Annette Sinclair, A general solution for a class of approximation

problems .................................

George Szekeres and Amnon Jakimovski, $(C, \infty)$ and $(H, \infty)$ methods of summation...................................... 867

Hale Trotter, Approximation of semi-groups of operators. ............. 887

L. E. Ward, A fixed point theorem for multi-valued functions ........... 921

Roy Edwin Wild, On the number of lattice points in $x^{t}+y^{t}=n^{t / 2} \ldots \ldots .929$ 